\title{
A RELAÇÃO ENTRE PRESSÃO AMBIENTAL E COMPORTAMENTO ESTRATÉGICO: UMA PESQUISA EM MPE DO SETOR DE AUTOPEÇAS
}

\section{RESUMO}

Este estudo teve como objetivo analisar a relação entre pressões ambientais e comportamento estratégico de MPE pertencentes a um agrupamento comercial de autopeças. A pesquisa, de abordagem quantitativa e com finalidade descritiva foi conduzida por meio de uma survey respondida por 32 empresas de forma satisfatória. Os resultados indicaram a predominância da estratégia genérica "analítica", composta por pouco mais da metade da amostra de empresas, em segundo lugar a estratégia "defensiva" e por último, um número menor de empresas com estratégias "reativas", de acordo com a classificação de comportamento estratégico de Miles e Snow. Uma análise de conglomerado efetuada a partir das pressões ambientais, às quais estão sujeitas as empresas, resultou no agrupamento das mesmas em três grupos. Por sua vez, os resultados tanto do teste do qui-quadrado quanto da análise discriminante confirmatória não evidenciaram uma forte relação de dependência entre estratégias competitivas adotadas pelas empresas pesquisadas e pressões ambientais. Em certa medida, a conclusão adotada corrobora a teoria, já que o universo pesquisado (pequenas e micro empresas com grau leve a moderado de maturidade), aparentemente não seria o terreno mais fértil para a aplicação dos pressupostos da escola ambiental de estratégia. Outra possível explicação para este resultado, também apoiada na teoria, é o fato de, mesmo quando suas empresas estão sujeitas a pressões ambientais similares, cada dirigente pode interpretar o ambiente de forma individualizada, conforme sugere a abordagem cognitiva de estratégia.

Palavras-chave: Pressão Ambiental; Escola Ambiental; Teoria Institucional; Comportamento Estratégico; Micro e Pequenas Empresas.

\section{THE RELATIONSHIP BETWEEN ENVIRONMENTAL PRESSURE AND STRATEGIC BEHAVIOR: A RESEARCH ON SME OF THE AUTO PARTS SECTOR}

\section{ABSTRACT}

This study aimed to analyze the relationship between environmental pressures and strategic behavior of MSE belonging to a trade group for auto parts. Results of the survey conducted - and satisfactorily answered by 32 companies showed the prevalence of the "analytical" generic strategy, composed of little more than half the sample, followed by the "defensive" strategy and finally, a smaller number of companies with "reactive" strategies according to the classification of strategic behavior of Miles and Snow. A cluster analysis effected from environmental pressures to which the companies are subject resulted in their grouping into three groups. In turn, the results of both the chi-square test as the confirmatory discriminant analysis did not show a strong dependency relationship between competitive strategies adopted by the companies surveyed and environmental pressures. To some extent, this conclusion corroborates the theory, since the universe surveyed (small and micro enterprises with mild to moderate degree of maturity), apparently is not the most fertile ground for the application of the conditions of the school's environmental strategy. Another possible explanation for this result also supported by the theory is that, even when their companies are subject to similar environmental pressures, each leader may interpret the environment individually, as suggested by the cognitive approach to strategy.

Keywords: Environmental Pressure; Environmental School; Institutional Theory; Strategic Behavior; Micro and Small Enterprise. 


\section{LA RELACIÓN ENTRE LA PRESIÓN AMBIENTAL Y EL COMPORTAMIENTO ESTRATÉGICO: UNA INVESTIGACIÓN SOBRE MICRO Y PEQUEÑAS EMPRESAS DEL SECTOR DE PIEZAS DE AUTOMÓVIL}

\section{RESUMEN}

Este estudio tuvo como objetivo analizar la relación entre las presiones medioambientales y el comportamiento estratégico de las micro y pequeñas empresas que pertenecen a un grupo de comercio de autopartes. La investigación, de enfoque cuantitativo y con el propósito descriptivo se llevó a cabo a través de una encuesta respondida por 32 empresas de manera satisfactoria . Los resultados indican el predominio de la estrategia " analítica " genérica , que consta de poco más de la mitad de la muestra de empresas, en segundo lugar, la "defensiva " y, en último , un menor número de empresas con estrategia "reactivas", de acuerdo con la clasificación de los comportamientos estratégicos de Miles y Snow . Un análisis de conglomerados realizado de las tensiones ambientales a las que están sujetas las empresas del mismo grupo se tradujo en tres grupos. A su vez, los resultados tanto de la chi-cuadrado como el análisis discriminante de confirmación no mostraron una fuerte relación de dependencia entre las estrategias competitivas adoptadas por las empresas encuestadas y presiones ambientales. Hasta cierto punto, la conclusión corrobora la teoría de que en el universo que se ha investigado ( micro y pequeñas empresas con la madurez moderada o leve), al parecer, no es el terreno más fértil para la aplicación de las condiciones de la escuela ambiental de estrategia. Otra posible explicación de este resultado, también apoyada en la teoría, es que, aun cuando sus empresas están sujetas a las presiones ambientales similares, cada gerente puede interpretar el medio ambiente de forma individual, como lo sugiere el enfoque de la estrategia cognitiva.

Palabras clabe: Presión Ambiental; Escuela Ambiental; La Teoría Institucional; Comportamiento Estratégico; Micro y Pequeñas Empresas.

\footnotetext{
${ }^{1}$ Mestre em Administração pela Universidade Estácio de Sá - UNESA. Professor e coordenador da Universidade Estácio de Sá - UNESA. Brasil. E-mail: dimasvidal@yahoo.com.br

${ }^{2}$ Doutor em Administração pela Universidade Federal do Rio de Janeiro - UFRJ. Professor da Universidade Estácio de Sá - UNESA. Brasil. E-mail: jose.geraldo@ estacio.br

${ }^{3}$ Doutor em Administração pelo Instituto Coppead de Administração da Universidade Federal do Rio de Janeiro UFRJ. Professor da Universidade Estácio de Sá - UNESA. Brasil. E-mail: $\underline{\text { marco.bouzada @estacio.br }}$
} 


\section{INTRODUÇÃO}

Para Mintzberg, Ahlstrand e Lampel (2007), o ambiente externo de uma organização compreende os relacionamentos da organização com fornecedores, clientes, agências governamentais, agências reguladoras, concorrentes etc. Ao longo do tempo tais relacionamentos produzem um conjunto cada vez mais complexo e poderoso de normas que dominam a prática. Para ter sucesso, uma organização precisa satisfazer e dominar estas normas. Com o tempo, isto leva as organizações que estão no mesmo ambiente a adotarem estruturas e práticas semelhantes, gerando uma situação conhecida como isomorfismo institucional. Este fenômeno tem sido utilizado com frequência para explicar a formação da estratégia como um processo de adaptação às pressões ambientais. Pesquisadores alinhados com esta abordagem enfatizam que as ações de estruturação organizacional são revestidas de significados sociais, com base em valores socialmente construídos, cujo propósito maior é obter legitimidade ou conformidade às normas e aos padrões institucionais estabelecidos. (DIMAGGIO; POWELL, 1983, 2005; OLIVER, 1991, 1992; SCOTT, 2001; FONSECA, 2003; MACHADO-DA-SILVA; COCHIA, 2004; CRUBELLATE; FONSECA; MACHADO-DA-SILVA, 2005; MACHADO-DASILVA; FONSECA, 2010) Entretanto, estes mesmos pesquisadores alertam para o fato de que o ambiente externo da organização é interpretado pelo estrategista a partir de valores pessoais individuais e esquemas mentais adquiridos a partir de sua experiência passada. Como explicar o fato de que duas organizações podem operar com sucesso no mesmo ambiente com estratégias diferentes? Ou seja, poder-se-ia afirmar que elas não reagiram da mesma forma às pressões ambientais.

Para estes autores, portanto, a necessidade de

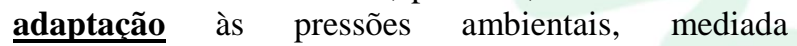
(moderada) por uma interpretacão individual do ambiente organizacional, juntamente com fatores de natureza econômica, poderiam influenciar, condicionar ou mesmo determinar, as escolhas de estratégias competitivas de uma organização.

Neste contexto, a presente pesquisa teve como objetivo avaliar, em micro e pequenas empresas pertencentes ao agrupamento comercial de autopeças da cidade fluminense de Nova Iguaçu, a relação entre pressão ambiental e as estratégias competitivas utilizadas por estas empresas. Para essa finalidade, foi necessário classificar as estratégias competitivas das empresas pesquisadas, identificar as fontes de pressão ambiental a que elas são submetidas, assim como sua intensidade e, finalmente, determinar a relação entre as intensidades das pressões ambientais e as estratégias identificadas.

Em face do importante papel representado pelas MPE na economia do país, os resultados da presente pesquisa poderão contribuir para uma melhor compreensão do processo de escolhas de estratégias competitivas por micro e pequenas empresas de varejo, a partir da população pesquisada, bem como fornecer subsídios para políticas governamentais de desenvolvimento de MPE.

\subsection{Hipótese de Pesquisa}

Existe uma forte relação entre a pressão ambiental e as estratégias competitivas utilizadas pelas empresas do agrupamento comercial pesquisado.

\section{REFERENCIAL TEÓRICO}

\subsection{Estratégias Competitivas}

Ao longo das últimas décadas o tema estratégia competitiva tornou-se um dos temas mais estudados e discutidos, tanto no meio acadêmico, quanto no meio empresarial. O cenário econômico competitivo dos países desenvolvidos no século passado e o acirramento deste cenário no início deste século, com a entrada agressiva dos países emergentes, estabeleceu a condição adequada para o desenvolvimento de modelos de estratégias genéricas (ANSOF, 1965; PORTER, 2003; MINTZBERG et al, 2003), amplamente utilizado por diferentes organizações.

Miles e Snow (1978) classificam as estratégias competitivas em quatro tipos: prospectora, defensiva, analítica e reativa, conforme definidas na Figura 1. As três primeiras se caracterizam por enfatizarem uma adequada coerência entre ambiente, estrutura e ação, pois as escolhas estratégicas estariam alinhadas aos processos e à estruturação organizacional, e estas com a primeira. A última, considerada não-estratégia, se caracteriza pelo não-alinhamento entre ações, processo e estrutura. 
A Relação entre Pressão Ambiental e Comportamento Estratégico: Uma Pesquisa em MPE do Setor de Autopeças

\begin{tabular}{|c|l|}
\hline $\begin{array}{c}\text { CATEGORIA } \\
\text { ESTRATÉGICA }\end{array}$ & \multicolumn{1}{c|}{ CARACTERÍSTICAS } \\
\hline Estratégia prospectora & $\begin{array}{l}\text { A empresa está continuamente ampliando a sua linha de produtos/serviços. Enfatiza a importância de } \\
\text { oferecer novos produtos/serviços em uma área de mercado relativamente ampla. Valoriza ser uma } \\
\text { das primeiras a oferecer novos produtos, mesmo que todos os esforços não se mostrem altamente } \\
\text { lucrativos. }\end{array}$ \\
\hline Estratégia analítica & $\begin{array}{l}\text { A empresa tenta manter uma linha limitada de produtos/serviços relativamente estável e, ao mesmo } \\
\text { tempo, adicionar um ou mais produtos/serviços que foram bem sucedidos em outras empresas do } \\
\text { setor. Em muitos aspectos, é uma posição intermediária entre as estratégias prospectora e defensiva. }\end{array}$ \\
\hline Estratégia defensiva & $\begin{array}{l}\text { A empresa procura localizar uma linha de produtos/ serviços ou mercados estáveis. O seu foco se } \\
\text { concentra em uma gama de produtos/serviços mais limitada do que seus concorrentes e tentam } \\
\text { proteger seu domínio por meio de oferta de produtos com melhor qualidade, serviços superiores e/ou } \\
\text { menores preços. Não procura estar entre os líderes de uma indústria, restringindo-se àquilo que sabe } \\
\text { fazer melhor que seus concorrentes. }\end{array}$ \\
\hline Estratégia reativa & $\begin{array}{l}\text { A empresa exibe comportamento mais inconsistente do que os outros tipos. Ė uma espécie de não- } \\
\text { estratégia. Não arrisca em novos produtos/serviços, a não ser quando ameaçados por competidores. A } \\
\text { abordagem típica é "esperar para ver" e responder somente quando forçada por pressões } \\
\text { competitivas, para evitar a perda de clientes e/ou manter a lucratividade. }\end{array}$ \\
\hline
\end{tabular}

Figura 1 - Estratégias competitivas de Miles e Snow Fonte: Miles e Snow (1978).

Miles e Snow (1978) sustentam que, apesar de as condições ambientais afetarem ou limitarem as escolhas estratégicas, elas não seriam capazes de determiná-las, pelo menos não para todos os atores envolvidos. Ghobril e Moori (2007), referenciando Miles e Snow (1978), destacam que, apesar da influência dos fatores ambientais no processo de estruturação organizacional, estes não eliminariam a capacidade de agência, que, segundo Crubellate, Fonseca e Machado-da-Silva (2005), é a capacidade de uma organização, através de seus dirigentes responsáveis pela estruturação organizacional, de interferir em eventos e produzir resultados, de forma intencional ou não. Portanto, objetivos e valores pessoais e sociais envolvidos no processo de decisão constituiriam fatores igualmente importantes, responsáveis pelo comportamento organizacional, que levariam a escolhas estratégicas.

Machado-da-Silva e Cochia (2004) afirmam que uma posição estratégica se estabelece quando há consistência entre as ações estratégicas (organizacionais) e a disposição de recursos, de produtos/serviços e de mercados ao longo de um período, em função de objetivos que garantam a sobrevivência e a competitividade de uma organização. Para Hitt et al. (2008), as ações estratégicas (organizacionais) ao contrário das ações táticas, são aquelas que envolvem um comprometimento significativo de recursos e são difíceis de serem implantadas e revertidas.

Para Miles e Snow (1978) as organizações desenvolvem um padrão de comportamento relativamente estável de escolhas estratégicas em função de uma combinação de fatores ambientais: o mercado, a tecnologia, o governo, e a relação com fornecedores. Estes fatores tendem a influenciar a estruturação organizacional, e ajudam a responder três problemas relacionados: 1) o problema empreendedor ou empresarial, com a busca de um domínio de um mercado ou produto, 2) o problema de engenharia, com a escolha e alocação de recursos que constituirão seu modelo tecnológico, e 3) o problema administrativo, relacionados aos processos gerenciais e organizacionais necessários. Teixera, Soares e Pelissari (2011) enfatizam que, em função do problema empreendedor, a organização decidirá alocar recursos significativos para atingir objetivos de natureza empreendedora ou empresarial. Afirmam ainda que deva haver um comprometimento de recursos de produção e de distribuição como resposta às questões relacionadas ao problema de engenharia e/ou modelo tecnológico, e por fim, a formulação e implementação de processos gerenciais e operacionais como solução aos problemas administrativos.

Diversas pesquisas que adotaram a taxonomia de Miles e Snow (1978) mostraram resultados que confirmam sua adequação para classificar padrões de comportamento estratégico de empresas dentro da realidade brasileira (GIMENEZ et al., 1999; VASCONCELOS; GUEDES; CANDIDO, 2007; MARTINS et al., 2008; LOPES; MOURA; OLIVEIRA, 2010; ALMEIDA; ANTONIALLI; ALMIRALVA, 2010; RIBEIRO, ROSSETTO, VERDINALLI, 2011; TEIXEIRA; SOARES; PELISSARI, 2011). Com exemplo podem ser citados os resultados de pesquisa de Fagundes e Gimenez (2009) que mostraram que a estratégia mais aplicada por 70 MPEs industriais paranaenses foi a prospectora, que esteve presente em $48,6 \%$ das empresas. Em seguida, surgiu a defensiva, com $30,0 \%$, a analítica, com $18,6 \%$, e a menos adotada foi a reativa, com $2,9 \%$. A relação entre turbulência ambiental e estratégia foi 
também testada na pesquisa acima mencionada. Os resultados sugerem que a estratégia defensiva mostrase mais presente em ambientes não turbulentos enquanto a prospectora predomina em ambientes turbulentos. Esse resultado é consistente com o modelo teórico de Miles e Snow (1978), que argumentam sobre a predominância de empresas prospectoras em ambientes mais dinâmicos, e defensivas em ambientes mais estáveis. Por outro lado, a estratégia analítica mostrou-se uniformemente distribuída nos dois graus de turbulência.

\subsection{Relação entre Pressão Ambiental e Comportamento Estratégico}

Mintzberg, Ahlstrand e Lampel (2007) destacam a importância da escola ambiental para o pensamento estratégico, pois trouxe o ambiente para o centro de discussão. Não como o fazem as escolas do design, do planejamento e do posicionamento, visto que, para estas, o ambiente é o fator a ser conquistado e dominado. Para a escola ambiental, no entanto, o ambiente é o fator condicionante no processo de desenvolvimento de estratégias, tirando do estrategista este papel. Entretanto, os autores alertam que talvez o terreno mais fértil para a aplicação do pensamento desta escola seria aquele formado por organizações em situações de poucas opções estratégicas (submetidas a muitas restrições), como é o caso de organizações localizadas no estágio de maturidade (fase conservação) de seu ciclo de vida, situação esta representada usualmente pela grande presença de sunk costs.

Uma das mais importantes contribuições no desenvolvimento teórico da Escola Ambiental partiu da teoria institucional e, posteriormente, da teoria neoinstitucional. DiMaggio e Powell (2005) levantaram a seguinte questão: “[...] O que torna as organizações tão similares"? Neste questionamento, os autores destacaram que os atores econômicos, no uso da chamada racionalidade limitada, na medida em que buscam transformar suas organizações, acabam tornando-as cada vez mais similares.

Fonseca (2003) afirma que a principal contribuição do neo-institucionalismo à teoria organizacional e seu desdobramento no pensamento estratégico é a ênfase da influêància do ambiente, ao colocar a legitimidade e a conformidade como fatores determinantes para a sobrevivência das organizações. Segundo este autor, a teoria institucional está baseada em três pilares institucionais que delineiam as relações entre as organizações e as instituições: os pilares regulador, normativo e cognitivo. Para Hashimoto e Fonseca (2009), empresas expostas ao mesmo conjunto de fontes de pressão ambiental, derivados dos 3 pilares, acabam por adotar ações e estruturas similares, fenômeno denominado de isomorfismo institucional. Ele se subdivide em 3 tipos: isomorfismo coercitivo, relacionado com o pilar regulador, isomorfismo mimético, relacionado com o pilar cognitivo, e isomorfismo normativo, relacionado com o pilar normativo.

\subsubsection{Isomorfismo coercitivo}

Para Fonseca (2003), o pilar regulador representa o conjunto de fontes de pressão ambiental relacionadas a regras, sanções ou à legitimidade, cuja resposta é definida como isomorfismo coercitivo. Segundo DiMaggio e Powell (2005) o isomorfismo coercitivo resulta de pressões por conformidade, formais ou não, exercidas por instituições ligadas ao estado, responsáveis pelo estabelecimento de leis, normas e procedimentos, instituições normativas privadas ligadas ao estabelecimento de padrões ou, ainda, por outras organizações empresariais das quais dependem direta ou indiretamente. Ainda segundo DiMaggio e Powell (2005), pressões ambientais do tipo coercitivo geram comportamento de busca por conformidade na tentativa de garantir legitimidade à organização junto ao estado, aos fornecedores, aos clientes, aos colaboradores, ou seja, junto à sociedade como um todo.

Para Hrebiniak (2005), uma organização, ao estabelecer uma estratégia institucional, em sua essência não tem como objetivo principal a obtenção de vantagem competitiva, e sim a busca pela preservação e pela estabilidade obtida pela conformidade a regras e a padrões institucionais coercitivos. A adequação às normas e às regras socialmente aceitas ou impostas leva as organizações à adoção de estratégias equivalentes, buscando desenvolver habilidades e competências em respostas a estas pressões ambientais, resultando em estruturas organizacionais homogêneas (HASHIMOTO; FONSECA, 2009).

Fagundes e Gimenez (2009) destacam que em relação às micro e pequenas empresas, dois fatores recentes têm contribuído para mudanças no papel do governo como fonte de pressão. A primeira é a lei Geral da Micro e Pequena Empresa, mais conhecida como Super Simples, unificando os diversos impostos que incidiam sobre elas e padronizando os instrumentos de escrituração. A segunda é a obrigatoriedade, por parte das médias e grandes empresas, da emissão da nota fiscal eletrônica. Aparentemente, o primeiro fator contribui para a diminuição de pressões ambientais pela simplificação e pela desburocratização dos processos fiscais e tributários, enquanto o segundo implica em um aumento de pressões ambientais pelo aumento da capacidade de fiscalização por parte do governo, diminuindo as possibilidades de sonegação por parte das micro e pequenas empresas de varejo, visto que estas são geralmente dependentes de fornecedores de empresas de médio e grande porte. 


\subsubsection{Isomorfismo mimético}

O pilar cognitivo, segundo Fonseca (2003), representa a maneira como os indivíduos responsáveis pelas decisões nas organizações percebem e interpretam as pressões relacionadas à incerteza e à ambiguidade de metas, respostas estas culturalmente sustentáveis, socialmente justificadas, onde a busca por legitimidade representa a essência do comportamento organizacional. A resposta a estas pressões recebe a denominação de isomorfismo mimético.

No isomorfismo mimético, as pressões ambientais relacionadas à incerteza, principalmente quando em um ambiente competitivo, compõem forças poderosas, que encorajam a imitação e a repetição de estratégias consideradas bem sucedidas. (DIMAGGIO; POWELL, 2005; HAYASHI; GIMENES; GRAVE, 2007). Ainda segundo estes autores, o comportamento mimético apresenta vantagens significativas, quando comparadas com possíveis alternativas, em termos de economia de recursos aplicados em sua formulação e facilidade de aplicação, principalmente em um cenário de incerteza e ambiguidades de metas.

Para Bataglia, Silva e Klement (2011) o processo de imitação parte da tentativa de copiar as competências de concorrentes que levaram a estratégias bem sucedidas. Para Simon (1970) em seu modelo de formação do processo decisório, limitações cognitivas se traduzem em decisões limitadas racionalmente. Para Machado-da-Silva, Fonseca e Fernandes (1998), longe de ser um processo racional perfeito, o processo de decisão estratégica representa uma resposta às demandas ambientais em função das limitações racionais, a incerteza e da ambiguidade de objetivos, podem levar as organizações a adotarem estratégias mais fáceis e rápidas de serem implementadas, gerando um processo de imitação das estratégias consideradas bem sucedidas.

\subsubsection{Isomorfismo normativo}

O pilar normativo representa o conjunto de fontes de pressão ambiental relacionadas à profissionalização da gestão, ao desenvolvimento de padrões e modelos de administração estratégica desenvolvidos pela atividade acadêmica ou por conceituadas empresas de consultoria; ou ainda representa o poder de influência das associações empresariais na adoção destes padrões estabelecidos. A conformação a estas fontes de pressão gera o isomorfismo normativo (FONSECA, 2003).

Para DiMaggio e Powell (2005), o isomorfismo normativo está relacionado às respostas padronizadas a pressões normativas, principalmente ligadas à profissionalização, ou à disseminação e institucionalização de práticas formais de gestão.

Machado-da-Silva e Vizeu (2007) destacam a importância e influência da produção acadêmica nas práticas empresariais e estas, por sua vez, representam o cenário onde são observados e desenvolvidos os conceitos que vão fundamentar a teoria. Nestes termos, a produção acadêmica influencia e é influenciada pela prática empresarial.

DiMaggio e Powell (2005) destacam a formação e o desenvolvimento das redes de profissionais, pelas quais se disseminam estes modelos e práticas para além das fronteiras das organizações. Outros dois mecanismos de pressão que estimulam o isomorfismo normativo são originados por meios das associações de classes profissionais e/ou empresariais e pelos mecanismos envolvidos no processo de seleção de pessoal, quase sempre oriundos das mesmas universidades e dos mesmos cursos de pós-graduação e de MBA.

Neste contexto, estas fontes de pressão normativa tendem a estabelecer a necessidade de legitimidade e de conformidade a práticas formais de estratégias, a práticas gerenciais cerimoniais, não necessariamente aquelas relacionadas à busca por bons resultados gerenciais e operacionais. (MACHADODA-SILVA; VIZEU, 2007)2.2.4 O Processo da reconstrução institucional

Diversas são as críticas à nova teoria institucional. No entanto, as maiores críticas surgiram exatamente dos próprios neo-institucionalistas ou como sugerem Crubelatte et al. (2005), daqueles que a utilizam como interface com outras linhas de pensamento. Alguns destes autores buscam uma visão multipragmática, onde os fenômenos da institucionalização e da desinstitucionalização são construídos a partir do mesmo conjunto de forças e que fazem parte das construções sociais (OLIVER, 1991; OLIVER, 1992; MACHADO-DA-SILVA; COCHIA, 2004; CRUBELATTE et al., 2005). Para estes autores, as fontes de pressão ambiental relacionadas às mudanças institucionais, a partir da desinstitucionalização, são tão importantes quanto aqueles que promovem o isomorfismo institucional.

Machado-da-Silva e Cochia (2004) e Crubelatte et al. (2005) destacam o papel de agência, no tocante à institucionalização de novas práticas organizacionais, juntamente com os valores sociais institucionalizados, como fundamentais no processo, tanto da persistência como de mudanças de valores sociais institucionalizados. Crubelatte et al. (2005), a partir de Oliver (1992), destacam que são três as possibilidades do atendimento à conformidade e à busca por legitimidade a partir da percepção e da interpretação de pressões institucionais: $1^{\circ}$ ) A reconstrução institucional e a busca pela legitimidade através da criação de novos valores sociais, como mais um recurso estratégico a ser obtido pela organização. $\left.2^{\circ}\right) \mathrm{O}$ atendimento à conformidade e à busca por legitimidade como um fator interveniente e limitante de escolhas de estratégias competitivas e $3^{\circ}$ ) $\mathrm{O}$ atendimento da conformidade e a busca por 
legitimidade como fator estruturado e estruturante da definição da própria estratégia.

Ainda segundo Crubelatte et al. (2005), no primeiro caso, a partir do questionamento dos valores sociais vigentes, contrário aos interesses de grupos, poderão ser geradas pressões que impossibilitem a reprodução de padrões institucionalizados, ocasionando a perda de legitimidade, desencadeando processo de desinstitucionalização e a possibilidade da construção de novos padrões e valores socialmente aceitos. Os atores envolvidos irão disseminar tais práticas e padrões para que se transformem em recursos com o objetivo de obterem vantagens competitivas. No segundo caso, os demais atores, a partir da interpretação das mudanças ambientais e da institucionalização de novas normas e práticas, terão que rever e adaptar suas opções estratégicas ao aumento das pressões institucionais. Por último, aqueles com menos recursos e mais suscetíveis às pressões institucionais, quando da alta presença de isomorfismo institucional serão forçados a atender às conformidades e a adotar práticas institucionalizadas como garantia de sobrevivência. Os autores ainda sugerem uma maior atenção aos fatores presentes no processo de desinstitucionalização, denominados por Oliver (1992) de fatores antecedentes ao processo de institucionalização.

Mais que uma crítica aos fundamentos do novo institucionalismo, Oliver (1991, 1992) sugere uma visão antecedente às fontes de pressão ambiental sugeridas por DiMaggio e Powell (2005), no sentido de completar as lacunas existentes na nova teoria institucional, cujo propósito final é compreender melhor o processo de homogeneização das estruturas organizacionais, denominadas por este último de isomorfismo institucional. Oliver (1992) enumera três grupos de fontes de pressão ambiental que estariam presentes na construção e/ou reconstrução de valores institucionalizados: pressões de natureza instrumental, pressões de natureza política e pressões de natureza social. Machado-da-Silva e Cochia (2004) e Crubelatte et al. (2005) destacam que este conjunto de fontes de pressão ambiental, divididos em três categorias, podem ser formados por subconjuntos de fontes que podem estar relacionados com o processo de mudanças, enquanto outros poderiam estar relacionados à estabilidade e à conformidade institucional.

Segundo Oliver (1992), as pressões instrumentais estão relacionadas às condições técnicas ou econômicas que comprometem ou levantam dúvidas sobre a legitimidade de valores institucionalizados ou quando resultados instrumentais para a conformidade são questionados, ou ainda quando fatores dissonantes aos valores compartilhados desestabilizam aqueles institucionalizados. As pressões de natureza política decorrem das crises de desempenho, da perda da eficiência e da diminuição da competitividade, da necessidade em adotar novas práticas gerenciais e da coalizão de forças que se mobilizam por mudanças, ou ainda das mudanças exigidas pelo ambiente para se obter legitimidade ou conformidade aos padrões institucionais ora estabelecida. Finalmente, as pressões de natureza sociais decorrem do enfraquecimento dos valores institucionalizados, do surgimento de novos significados decorrentes de conflitos sociais, das mudanças de comportamento e da construção de novos valores sociais compartilhados.

Esta seção se fecha com a proposta, na Figura 2 , de fontes de pressão ambiental agrupadas em 6 categorias. Estas fontes foram retiradas do referencial teórico de apoio a esta pesquisa e ainda tomando como referência o modelo de identificação de fontes de pressão ambiental desenvolvido por Fernandes et al. (2009).

\begin{tabular}{|c|l|}
\hline CÓDIGO & \multicolumn{1}{c|}{ FONTES DE PRESSÃO COERCITIVA } \\
\hline C1 & Ações do governo através de suas agências regulatórias e instrumentos de fiscalização. \\
\hline C2 & Adequação aos instrumentos de arrecadação tributária. \\
\hline C3 & $\begin{array}{l}\text { Existência de poucos e grandes fornecedores de produtos/serviços para as organizações do setor (máquinas e } \\
\text { equipamentos, matérias primas e insumos). }\end{array}$ \\
\hline C4 & Forte dependência de uma empresa a um ou poucos fornecedores de recursos vitais ou estratégicos. \\
\hline M1 & $\begin{array}{l}\text { AmoNTES DE PRESSÃO MIMÉTICA } \\
\text { organizações que ela considera bem sucedidas. }\end{array}$ \\
\hline M2 & $\begin{array}{l}\text { Incertezas sobre a adequação dos meios (estrutura organizacional da empresa) aos seus fins (objetivos estratégicos) } \\
\text { fazendo com que a organização procure se moldar em outras organizações que ela considera bem sucedidas. }\end{array}$ \\
\hline M3 & Pequeno número de alternativas visíveis de modelos de negócio adequados ao setor. \\
\hline
\end{tabular}


A Relação entre Pressão Ambiental e Comportamento Estratégico: Uma Pesquisa em MPE do Setor de Autopeças

\begin{tabular}{|c|c|}
\hline & FONTES DE PRESSÃO NORMATIVA \\
\hline N1 & Crescente profissionalização das atividades de gestão das empresas do setor. \\
\hline \multirow[t]{2}{*}{ N2 } & Adoção de padrões ou práticas gerenciais sugeridas por associações de classe ou cooperativas empresariais. \\
\hline & FONTES DE PRESSÃO INSTRUMENTAL OU FUNCIONAL \\
\hline I1 & Diminuição de preços e redução de custos no setor. \\
\hline $\mathbf{I}$ & Diminuição da rentabilidade pelo aumento de competição no setor. \\
\hline $\mathbf{I 3}$ & Necessidade crescente de capital de giro. \\
\hline I4 & Necessidade crescente de inovação em processos, produtos ou serviços. \\
\hline I5 & Reestruturação de instalações para adequação às mudanças em seu setor. \\
\hline \multirow[t]{2}{*}{ I6 } & Necessidade crescente de parcelamento de compras de consumidores. \\
\hline & FONTES DE PRESSÃO POLÍTICA \\
\hline P1 & Aumento da carga tributária. \\
\hline $\mathbf{P 2}$ & Escândalos políticos e crises econômicas nacionais e internacionais. \\
\hline \multirow[t]{2}{*}{$\mathbf{P 3}$} & Alta informalidade das empresas do setor. \\
\hline & FONTES DE PRESSÃO SOCIAL \\
\hline S1 & Ações de responsabilidade social relacionadas ao ambiente de trabalho. \\
\hline S2 & Atendimento aos direitos do consumidor. \\
\hline S3 & Presença alta de nichos (especialização) em seu mercado de atuação. \\
\hline S4 & Satisfação e fidelização do cliente. \\
\hline
\end{tabular}

Figura 2 - Fontes de pressão ambiental

Fonte: Elaboração própria, a partir de DiMaggio e Powell ( 2005), Fernandes et al. (2009), e Oliver (1992)

\section{METODOLOGIA}

A presente pesquisa é descritiva com abordagem quantitativa. Teve como instrumento de levantamento dos dados um questionário fechado contendo 5 questões para dados demográficos, 11 questões destinadas à classificação de comportamento estratégico, a partir do modelo adaptado por Ribeiro, Rossetto e Verdinelli (2011) do proposto por Conant, Mokwa e Varadarajan (1990), modelo este validado nas pesquisas de Gimenez et al. (1999), de Perola e Gimenez (2000), de Martins et al. (2008), de Lopes, Moura e Oliveira (2010), de Almeida, Antonialli e Amiralda (2010), de Teixeira, Soares e Pelissari (2011) e de Ribeiro, Rossetto e Verdinelli (2011). Para os dados referentes às fontes de pressão ambiental, o questionário continha 22 questões, uma para cada fonte de pressão listada na Figura 2, constituída de uma escala Likert com cinco opções, que vão de (1) Discordo totalmente a (5) Concordo totalmente.
Antes de o questionário ter sua versão final disponibilizada, foi realizado pré-teste, para revisar termos e conceitos colocados, em 3 empresas do setor, convidadas para este fim.

Da população de todas as micro e pequenas empresas pertencentes ao agrupamento comercial de autopeças e acessórios de automóveis da cidade fluminense de Nova Iguaçu, 32 delas responderam satisfatoriamente o questionário, compondo, assim, a amostra da pesquisa.

O critério de classificação do comportamento estratégico a partir do modelo de Miles e Snow (1978) foi o mesmo adotado por Ribeiro, Rossetto e Verdinelli (2011) a partir de Conant, Mokwa e Varadarajan (1990). As opções do questionário foram organizadas em quatro alternativas, sendo que cada uma delas está relacionada a um dos tipos do modelo, colocadas aleatoriamente para que não se tornassem tendenciosas. A classificação foi realizada a partir do maior número absoluto de respostas em um tipo, das quatro 
disponíveis, e em caso de empate entre as alternativas foram adotados os seguintes critérios: havendo empate entre o comportamento defensivo, prospector e ou analista, o resultado é classificado como analista, visto que este último apresenta-se como um comportamento intermediário entre os comportamentos prospector e defensivo. No caso de o empate envolver o comportamento reativo, o comportamento estratégico será classificado como reativo.

Para a classificação das empresas pesquisadas quanto às suas percepções das pressões ambientais presentes ou por eles consideradas de maior magnitude foi realizada uma análise de conglomerado. Para a formação e validação final dos grupos (conglomerados), a cada agrupamento obtido pela Análise de Conglomerado, foi realizada análise de variância dos grupos (ANOVA) até que se obtivessem grupos com diferenças estatísticas entre si. A partir da formação dos agrupamentos, estes passaram por uma Análise Discriminante confirmatória como um último processo de validação dos grupos obtidos.

A classificação das empresas quanto às pressões ambientais através da análise de conglomerado não se traduz em um instrumento que permita uma inferência de seus resultados. Para este fim, foi realizado um teste qui-quadrado para verificar se havia ou não diferença estatística entre a frequência das empresas classificadas pelo comportamento estratégico (MILES e SNOW, 1978) em cada conglomerado (definido em termos de pressão ambiental). Para tanto, se estabeleceu como hipótese nula não haver diferença estatística entre a frequência observada e a esperada (se não houvesse influência da pressão ambiental no comportamento estratégico, seria de se esperar que a frequência de empresas seguindo cada comportamento fosse a mesma em cada conglomerado). Como hipótese alternativa, haveria diferença estatística entre as duas frequências. Confirmando a hipótese nula, poder-se-ia afirmar que não existiria algum nível de correlação entre as variáveis comportamento estratégico e pressões ambientais, pois esta última não afetaria na composição da primeira.

Para garantir um maior índice de confiabilidade e uma possível confirmação dos resultados obtidos até esta fase, uma análise discriminante foi realizada pelo método stepwise de seleção de variáveis. Foi considerada como variável dependente o comportamento estratégico (categórica); as pressões ambientais foram consideradas como variáveis independentes. Como resultado, poder-se-ia obter uma função discriminante para o comportamento estratégico, tal que fosse possível estabelecer uma relação mais clara de causa e efeito entre este e as pressões ambientais.

Estes procedimentos estatísticos visaram a verificação da hipótese de pesquisa e o consequente atendimento do objetivo do trabalho.
Destaca-se como principal limitação metodológica da pesquisa o reduzido número de empresas que constituiu a amostra. Pelas dificuldades naturais do processo de coleta de dados, essa limitação, infelizmente, não pode ser contornada. Não obstante, acredita-se que a redundância almejada e obtida pela triangulação dos métodos utilizados venha a mitigar seus efeitos.

\section{APRESENTAÇÃO E ANÁLISE DOS RESULTADOS}

\subsection{Classificação do Comportamento Estratégico}

Os resultados da pesquisa mostraram que o agrupamento comercial de autopeças e acessórios da cidade fluminense de Nova Iguaçu é composto por micro e pequenas empresas cujos tempos de existência estão distribuídos de forma equilibrada em três grupos: empresas com menos de 5 anos de atividade, empresas com um período de 5 anos até 10 anos de existência e empresas que operam há mais de 10 anos. Estes dados sugerem que o agrupamento é formado basicamente por empresas de grau leve a moderado de maturidade .

Com relação ao comportamento estratégico, os resultados sugerem uma preferência das empresas pelo comportamento caracterizado como analítico, presente em pouco mais da metade das empresas pesquisadas. Um segundo grupo, composto por aproximadamente um terço das empresas pesquisadas, prefere o comportamento estratégico defensivo; e, finalmente um terceiro grupo, com menos de $20 \%$ das empresas, apresentou comportamento classificado como reativo. Nenhuma das empresas pesquisadas foi associada ao comportamento prospectivo.

Em certa medida, os resultados acima corroboram a teoria, já que seria esperado encontrar um número pequeno de empresas com comportamento prospectivo, visto se tratarem de micro e pequenas empresas. Este comportamento demanda posse de muitos recursos (MILES; SNOW, 1978, TEIXEIRA; SOARES; PELISSARI, 2011), o que não é o caso das empresas pesquisadas. Era de se esperar também pouco comportamento reativo, já que as empresas pesquisadas são usualmente dirigidas por micro e pequenos empresários, com características predominantemente empreendedoras (MINTZBERG; AHLSTRAND; LAMPEL, 2007).

A importância da posse de recursos para o comportamento prospectivo também é ressaltada nos resultados da pesquisa de Ribeiro, Rossetto e Verdinelli (2011). Ao aplicarem o modelo de estratégias de Miles e Snow (1978) para estabelecer uma relação entre estratégias e alocação dos recursos das empresas pesquisadas, de acordo com a teoria da Resource Based View (RBV), os autores constataram que os prospectores são aqueles que apresentaram a 
A Relação entre Pressão Ambiental e Comportamento Estratégico: Uma Pesquisa em MPE do Setor de Autopeças

maior correlação positiva, e que os reativos são os que apresentaram a maior correlação negativa entre escolhas estratégicas e posse de recursos.

Segundo Hrebiniak (2005), uma organização, ao estabelecer uma estratégia institucional, em sua essência não tem como objetivo principal a obtenção de vantagem competitiva, e sim a busca pela preservação e estabilidade obtida pela conformidade a regras e a padrões institucionais coercitivos. Isto justificaria o grande número $(50 \%)$ de empresas com comportamentos defensivos e reativos encontrado na pesquisa.
Finalmente, observa-se também que os resultados guardam semelhança com as pesquisas de Gimenez et al. (1999) envolvendo 107 pequenas empresas pesquisadas na cidade paranaense de Londrina, e de Almeida, Antonialli e Amiralva (2010,) conduzida em 75 pequenas empresas de propriedade ou de gestão feminina, na cidade mineira de Lavras. Nas duas pesquisas, verificou-se que o comportamento estratégico mais frequente foi o analítico.

\subsection{Formação de Conglomerados (Grupos) a Partir das Pressões Ambientais}

Depois da classificação do comportamento estratégico, os dados referentes às pressões ambientais passaram pela Análise de Conglomerado, através do SPSS 17. O dendograma resultante está apresentado na Figura 3 a seguir.

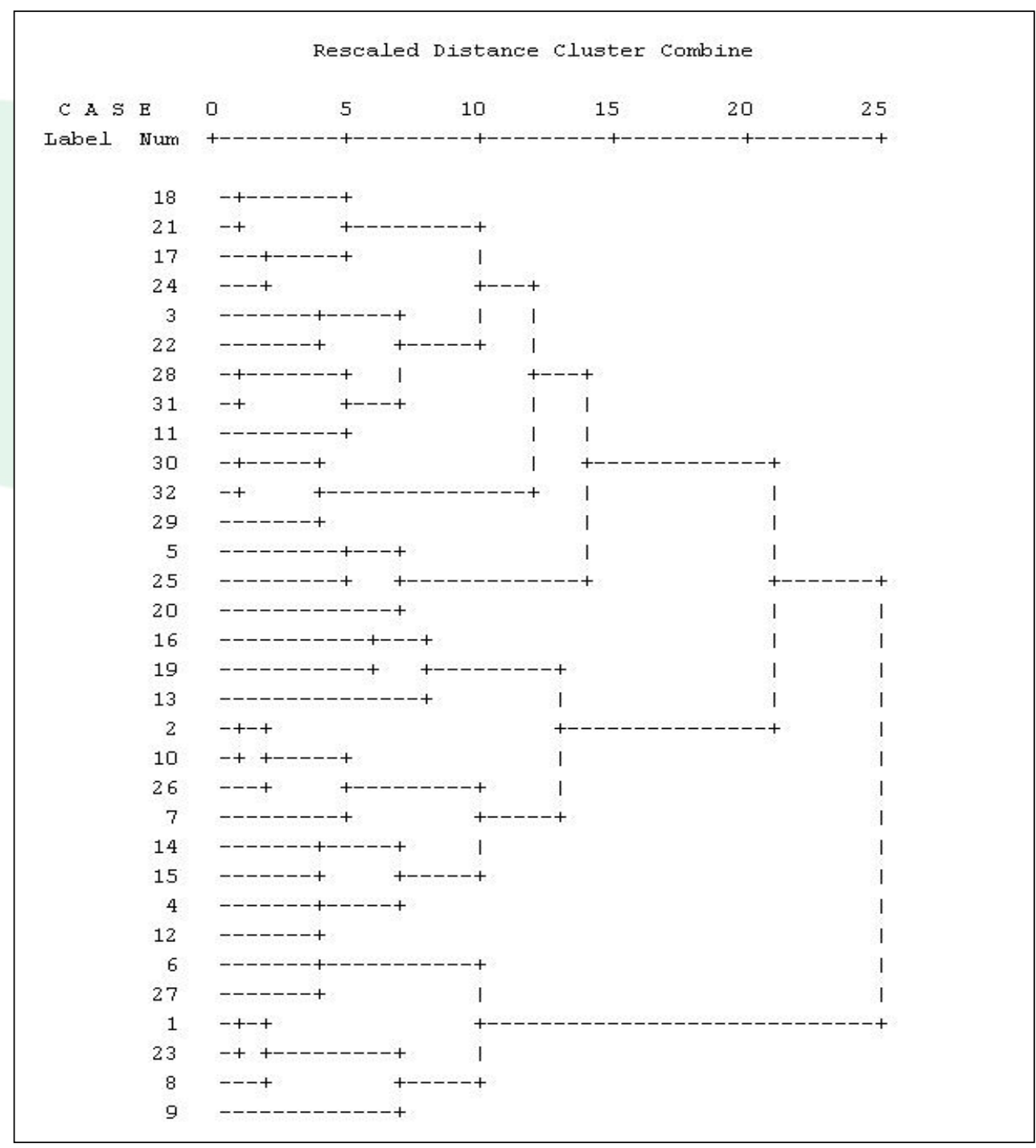

Figura 3 - Dendograma da Análise de Conglomerado Fonte: Elaborado pelos autores. 
Foram formados três grupos: um com 15, outro com 11 e outro com 6 empresas. A tabela 1 a seguir apresenta, para cada um dos 3 grupos formados, a média obtida em cada uma das variáveis (fontes de pressão ambiental). Foram descartadas as variáveis que obtiveram valor-p maior que 0,05 no teste de ANOVA, conforme sugerem Corrar et al. (2011).

Tabela 1 - Média de cada variável por grupo

\begin{tabular}{|c|c|c|c|}
\hline VARIÁVEIS & GRUPO 1 & GRUPO 2 & GRUPO 3 \\
\hline C3 & 4,07 & 2,45 & 1,67 \\
\hline C4 & 4,00 & 2,36 & 3,83 \\
\hline I & 4,27 & 4,36 & 2,00 \\
\hline I & 2,80 & 3,82 & 3,67 \\
\hline I6 & 3,60 & 4,36 & 1,83 \\
\hline M1 & 3,13 & 2,64 & 1,50 \\
\hline M3 & 3,40 & 2,55 & 1,67 \\
\hline N1 & 2,33 & 3,73 & 1,67 \\
\hline N2 & 2,67 & 1,18 & 1,33 \\
\hline S3 & 4,40 & 4,55 & 3,67 \\
\hline
\end{tabular}

Fonte: Elaborado pelos autores.

Principalmente os dois últimos grupos são bastante reduzidos, dificultando alguns testes estatísticos. Mas ainda assim é possível identificar diferenças estatisticamente significativas, apenas estas tendo que ser ainda mais evidentes em função do reduzido tamanho das amostras. Até por isso, conforme pode ser visto na tabela 1 anterior, nem todas as variáveis tiveram médias estatisticamente diferentes nos três grupos.

Adicionalmente, sabe-se que a média não é a medida mais adequada para lidar com variáveis ordinais (como as representadas por uma escala Likert), mas este indicador está sendo considerado para fornecer uma noção de magnitude.

Considerando um valor de média maior que 3 (correspondente a concordo ou concordo totalmente com a afirmativas do questionário), como um indicador da influência da fonte de pressão ambiental para as ações gerenciais, as variáveis, em cada grupo, ficariam distribuías conforme descrito nos parágrafos a seguir.

O grupo 3, formado por aproximadamente 19 $\%$ dos integrantes da amostra, pode ser considerado o grupo que menos sofre influência das fontes de pressão ambiental, pois apresenta somente 3 fontes de pressão ambiental (com médias maiores que 3) que afetam as ações gerenciais de suas empresas. Neste grupo, é possível dar um destaque para uma fonte coercitiva relacionada à dependência de fornecedores de recursos vitais (C4), e para uma fonte instrumental relacionada à inovação em processos, produtos ou serviços (I4). Este grupo é formado em sua maioria (66\%) por empresas com menos de 5 anos de atividades, confirmando suas características empreendedoras.

O grupo 2, formado por aproximadamente $34 \%$ das empresas da amostra, pode ser considerado como um grupo que sofre influência de um número moderado de fontes de pressão ambiental, com destaque para três fontes instrumentais relacionadas a: competição em preço (I1), parcelamento de vendas (I6) e inovação ( I4). É também o único grupo que sofre influência de fonte normativa, relacionado à profissionalização das atividades de gestão (N1). Dentre as variáveis levantadas na pesquisa, não havia nenhuma relacionada à formação profissional e/ou acadêmica dos empresários pesquisados, no entanto, no contato com estes ao longo do processo de coleta de dados, foi observado em alguns uma capacidade de articulação entre a teoria e a prática gerencial, evidenciando um domínio razoável sobre práticas padronizadas de gestão (FONSECA 2003, DIMAGGIO; POWELL (2005) .

Também foi possível constatar, por meio de observação ativa, a importância para alguns empresários do papel de associação de classe denominada Associação do Polo Automotivo de Nova Iguaçu como um centro de articulação política entre seus associados (HASHIMOTO; FONSECA, 2009). Essa constatação e a pressão ambiental relacionada à profissionalização das atividades de gestão (N1), mencionada no parágrafo anterior, colocam este grupo como aquele canditato à adoção de práticas gerenciais padronizadas (institucionalizadas) típicas do isomorfismo normativo (DIMAGGIO; POWELL, 2005).

Por fim, o grupo 1, formado por pouco menos da metade das empresas pesquisadas $(47 \%)$, é aquele que mais sofre influência de pressões ambientais, apresentando duas fontes de pressão ambiental coercitiva (C3 e C4), duas fontes de pressão ambiental instrumental (I1 e I6) e duas fontes de pressão ambiental mimética (M1 e M3). É importante destacar que foi o único grupo que apresentou fontes de 
isomorfismo mimético como fatores que afetam as ações gerenciais de suas empresas. Neste grupo, aproximadamente $53 \%$ de seus integrantes são compostos por empresas que atuam ha mais de 10 anos, confirmando em certa medida a teoria de que as organizações localizadas no estágio de maturidade de seu ciclo de vida, encontram-se na condição de poucas opções estratégicas, e portanto mais propícias ao isomorfismo institucional

(MINTZBERG; AHLSTRAND; LAMPEL, 2007).

Para confirmar o resultado obtido pela análise de Conglomerado, foi realizada uma Análise Discriminante confirmatória considerando como variável dependente os grupos obtidos e como variáveis independentes as variáveis com valor-p menor que 0,05 (CORRAR et al., 2011): C3, I6, C4, N1 E N2. Os resultados do teste de Wilk's Lambda indicaram que a função discriminante encontrada apresenta alta capacidade de discriminar os elementos entre os grupos (CORRAR et al., 2011). Neste caso, pode-se considerar como adequados os critérios de classificação dos grupos, tendo-se obtido $100 \%$ de acurária na classificação com os dados originais, 92,3 $\%$ dos dados cross-validated e 83,3\% dos dados da amostra de teste, confirmando a adequação na formação dos grupos pela Análise de Conglomerado.

A quantidade e a identificação, em cada grupo, das pressões ambientais relevantes (respostas nos três níveis mais elevados da escala de Likert), permitiu caracterizar as empresas de cada grupo:

- $\quad$ as empresas do Grupo 3 (agrupa as empresas sujeitas ao menor número de pressões ambientais relevantes e é o grupo com menor número de empresas) consideram que suas decisões estratégicas são pouco afetadas pelas pressões ambientais, e são as que mais se julgam "pressionadas a inovarem", especialmente no que concerne à inovação em processos e produtos;

- o Grupo 2 é formado por empresas que se consideram moderadamente afetadas pelas pressões ambientais, e caracterizam-se por serem mais propensas a adotarem padrões gerenciais padronizados a partir basicamente da pressão por "profissionalização da administração";

- e o Grupo 1 (agrupa as empresas sujeitas ao maior número de pressões ambientais relevantes, sendo o grupo com maior número de empresas) é formado por empresas que se consideram mais sujeitas às pressões ambientais, principalmente aquelas de natureza coercitiva e mimética.

\subsection{Relação entre Comportamento Estratégico e Pressões Ambientais}

As características das empresas de cada grupo, conforme listadas no último parágrafo da seção anterior, de certa forma confirmam a proposição de Oliver (1992) de que as empresas podem responder às pressões ambientais através de três modos:

- Utilização de reconstrução institucional e busca por legitimidade, através da criação de novos valores sociais, como um recurso estratégico competitivo (empresas do grupo $3)$.

- Aceitação da busca por conformidade e legitimidade como um fator interveniente e limitante de escolhas de estratégias competitivas (empresas do grupo 2).

- Aceitação da busca por conformidade e legitimidade como fator estruturado e estruturante de escolhas de estratégias competitivas (empresas do grupo 1).

Estes resultados se mostram ainda mais significativos, visto que todas as empresas pesquisadas atuam no mesmo setor econômico, na mesma região geográfica, e possivelmente afetados pelo mesmo conjuntos de pressões ambientais.

Consequentemente, a etapa seguinte consistiu em aplicar o teste Qui-quadrado para testar a dependência entre as variáveis Comportamento Estratégico e Pressões Ambientais. Conforme foi apresentado na Metodologia, o teste Qui-quadrado foi aplicado para verificar se havia ou não diferença estatística entre a frequência das empresas classificadas pelo comportamento estratégico (MILES; SNOW, 1978) em cada conglomerado (definido em termos de pressão ambiental). Para tanto foi estabelecido como hipótese nula a ausência de diferença estatística entre a frequência observada e a esperada. Neste caso, para se confirmar a não influência de pressão ambiental no comportamento estratégico classificado, esperava-se que a frequência das empresas seguindo cada comportamento fosse a mesma em cada conglomerado.

Os resultados do teste de Qui-quadrado mostraram que as variáveis Comportamento Estratégico e Pressões Ambientais são independentes, não havendo correlação significativa entre elas (ao nível de significância de 5\%). Mas este resultado merece ser visto como não conclusivo, porque alguns requisitos necessários para este tipo de teste ser totalmente confiável não foram alcançados, em função do reduzido número de empresas em alguns grupos. Reforça também a cautela acima mencionada o fato de que os resultados de pesquisa de Fagundes e Gimenez (2009) em 70 micro e pequenas empresas do Estado do Paraná, mostraram relação entre ambiente, no caso caracterizado pelo nível de turbulência, e estratégia 
competitiva adotada. De acordo com Fagundes e Gimenez (2009, p. 145), "a estratégia defensiva mostrou-se mais presente em ambientes menos turbulentos e a prospectora em ambientes mais turbulentos".

Em vista disso, tornou-se importante realizar a análise discriminante, antes definida como instrumento confirmatório, agora para tentar estabelecer as possíveis influências das pressões ambientais nas escolhas de estratégias competitivas das empresas pesquisadas. Nesta Análise Discriminante, foram consideradas como variáveis independentes todas as 22 variáveis de pressão ambiental levantadas pela pesquisa e como variável dependente a varável categórica, comportamento estratégico.

Os resultados da análise revelaram que, das 22 variáveis disponíveis, somente duas apresentaram valor-p menor que 0,05 , quando todas as variáveis foram consideradas simultaneamente, sendo as demais removidas da análise. As variáveis incluías foram: S3 Presença alta de nichos (especialização) em seu mercado de atuação (valor-p de 0,006); e M1 - Pressão causada pela Ambiguidade dos objetivos organizacionais (valor-p de 0,001).

O teste de Wilk's Lambda indicou que as funções encontradas têm um boa capacidade de discriminar os elementos entre os grupos; no entanto, apenas uma proporção moderada $(65,4 \%)$ dos casos de desenvolvimento da amostra e da amostra crossvalidated foram corretamente classificados, enquanto que houve acertos em apenas um terço da amostra de teste. Nestas condições, pode-se afirmar que o modelo tem um moderado nível de capacidade de classificação das empresas quanto ao comportamento estratégico.

\section{CONCLUSÕES}

Sobre as fontes de pressão ambiental, merecem ser destacados os baixos níveis de influência das fontes de pressão ambiental de natureza política e social sobre as ações gerenciais das empresas pesquisadas. Sobre as fontes de natureza política, acredita-se que a pequena influência da pressão "carga tributária" possa ser explicada pela criação de novos regimes tributários, como a substituição tributária (o Simples e o Super Simples), voltados especialmente para as micro e pequenas empresas, o que contribuiu também para a diminuição da pressão "Alta informalidade das empresas do setor".

Com relação às quatro fontes ambientais de natureza social, somente a "Presença alta de nichos (especialização) em seu mercado de atuação" obteve um nível alto de influência. Com relação às fontes: "Ações de responsabilidade social relacionadas ao ambiente de trabalho"; "Atendimento aos direitos do consumidor"; e "Satisfação e fidelização do cliente", não foi possível obter indícios que pudessem explicar seus baixos índices de influência nas iniciativas estratégicas das empresas.

Com relação ao objetivo da pesquisa, avaliar a relação entre pressão ambiental e estratégias competitivas destas empresas, os resultados do teste do Qui-quadrado, ainda que não conclusivos, mostraram que as frequências de comportamento estratégico (prospectora, analítica, defensiva e reativa) são similares nos três grupos de empresas definidos por meio da análise de conglomerados. Ou seja, os resultados sugerem ser pequena a influência das pressões ambientais sobre as escolhas de comportamento estratégico.

Como os resultados do teste Qui-quadrado foram não conclusivos, foi realizada uma análise discriminante para verificar se as 22 variáveis independentes (pressões ambientais) conseguiam discriminar a variável dependente categórica comportamento estratégico (prospectora, analítica, defensiva e reativa). Os resultados da análise discriminante apontaram para resultados ligeiramente diferentes, ou seja, para uma moderada evidência da influência de pressões ambientais nas escolhas de comportamento estratégico.

Levando-se em conta os resultados tanto do teste do Qui-quadrado quanto da análise discriminante, preferiu-se optar pela não evidência de relação forte entre pressão ambiental e escolhas estratégicas, no universo pesquisado. Em outras palavras, preferiu-se concluir pela pequena evidência de influência das pressões ambientais sobre a escolha de comportamento estratégico, não se confirmando a hipótese de pesquisa.

Convém deixar claro que essa conclusão não caracteriza uma limitação dos resultados da pesquisa, até porque são discutidas, a seguir, possíveis explicações para não ter sidoencontrada uma sólida influência das pressões ambientais sobre a escolha do comportamento estratégico.

Em certa medida, a conclusão adotada corrobora a teoria, já que o universo pesquisado (pequenas e micro empresas com grau leve a moderado de maturidade) não foi o terreno mais fértil para a aplicação dos pressupostos da escola ambiental de estratégia. Para esta escola, as organizações onde as iniciativas estratégicas são fortemente infuenciadas por pressões ambientais seriam aquelas em situação de poucas opções estratégicas, sob um conjunto grande de restrições, como é o caso de organizações localizadas no estágio de maturidade (fase conservação) de seu ciclo de vida, situação esta representada usualmente pela grande presença de sunk costs. Este não parece ser o caso das empresas pesquisadas.

Outra possível explicação para este resultado, também apoiada na teoria, é o fato de que, mesmo quando suas empresas estão sujeitas a pressões ambientais similares, cada dirigente pode interpretar o ambiente de forma individualizada, conforme preconiza a abordagem cognitiva de estratégia. Ou seja, 
mesmo quando duas empresas estão sujeitas a pressões ambientais similares, ainda assim seus dirigentes poderão adotar comportamentos estratégicos diferentes.

Como ideias para pesquisas futuras, sugere-se a obtenção de uma amostra maior, para que a limitação metodológica mencionada anteriormente seja eliminada. Adicionalmente, um trabalho semelhante a este, mas em outro setor da economia, poderia gerar resultados diferentes e contribuir para o arcabouço teórico acerca de Estratégia.

Finalmente, outros modelos de classificação de ambientes quanto à pressão - ver o artigo de Fagundes e Gimenez (2009) - e de empresas quanto ao seu comportamente estratégico - tipologias de estragégias genéricas de Porter (2004) e Mintzberg et al. (2007) - poderiam ser utilizados para proporcionar uma diversidade metodológica capaz de permitir generalizações mais confiáveis acerca dos resultados encontrados.

\section{REFERÊNCIAS}

Almeida, Ivana C., Antonialli, Luiz M., Gomes, Almiralva F. Comportamento Estratégico das Mulheres Empresárias: Um Estudo Baseado na Tipologia de Miles e Snow. Revista IberoAmericana de Estratégia - RIAE, São Paulo, v. 10, n. 1, p. 128-146, jan/abr. 2011.

Ansoff, Igor H. Corporate strategy. New York: McGraw-Hill, 1965.

Bataglia, Walter, SILVA, Adilson A. \& Klement, Claudia F. F. Dimensões da Imitação entre Empresas: Um Estudo na Indústria de Transformação Brasileira. Revista de Administração de empresas, São Paulo, v. 51, n. 2, 2011.

Cochia, Camila B. R. \& Machado-da-silva, Clovis L. Ambiente, Interpretação e Estratégia em Organizações Paranaenses dos Setores de Vestuário e Alimentos. Revista de Administração Contemporânea, Edição Especial, v.8, p. 11-35, 2004.

Conant, J. S.; Mokwa, M. P. \& Varadarajan, P. R. Strategic types, distinctive marketing competencies and organizational performance: a multiple measures-based study. Strategic Management Journal, v. 11, p. 365-383, 1990.

Corrar, Luiz J., Paulo, Edilson \& Dias Filho, José M. Análise Multivariada para os Cursos de Administração, Ciências Contábeis e Economia. São Paulo: Atlas, 2011.
Crubellate, João M., Fonseca, Valéria S. \& Machadoda-silva, Clovis. L. Estrutura, Agência e Interpretação: Elementos para uma Abordagem Recursiva do Processo de Institucionalização. RAC - Revista de Administração Contemporânea, Edição Especial. P. 09 - 39, 2005.

Dimaggio, Paul J. \& Powell, Walter W. A Gaiola de Ferro Revisitada: Isomorfismo Institucional e Racionalidade Coletiva nos Campos Organizacionais. RAE- Revista de Administração de Empresas, São Paulo, v. 45, n. 2, 2005.

Fagundes F.M.; Gimenez F. A. P. Ambiente, estratégia e desempenho em micro e pequenas empresas. REBRAE. Revista Brasileira de Estratégia, Curitiba, v. 2, n. 2, p. 133-146, maio/ago. 2009.

Fernandes, Bruno H. R., Graeff, Julia F., Angonese, Rosangela \& Nakatani, Márcia S. M. Pressões Ambientais, Stakeholders e Respostas Estratégicas: Proposição de Metodologia de Análise do Ambiente em Setores Produtivos. IV Encontro de Estudos em Estratégia, Anais... Recife, PE, 2009.

Fonseca, Valéria S. A Abordagem Institucional nos Estudos Organizacionais: Bases conceituais e Desenvolvimentos Contemporâneos. Segundo capítulo do livro Organizações, instituições e poder no Brasil. FGV, Rio de Janeiro, 2003.

Ghobril, Alexandre N. \& Moori, Roberto G. Alinhamento Estratégico entre Indústria de Bens de Capital e de Alimentos: Uma análise com Base em Miles e Snow. EnAnpad, Anais... Rio de Janeiro, 2007.

Gimenez, Fernando A. P., Pelisson, Cleufe, Kruger, Eugênio G. S. \& Hasyashi Jr, Paulo. Estratégia em Pequenas Empresas: Um a Aplicação do Modelo de Miles e Snow. Rac - Revista de Administração Contemporânea, v. 3, n. 2, p. 53-74, 1999.

Hayashi Jr, Paulo, Gimenez, Fernando A. P. \& Grave, Paulo S. Isomorfismo Mimético em Estratégia: uma ferramenta para investigação. Revista de Administração Mackenzie, v. 8, n. 4. 2007.

Hashimoto, Nelson Y. \& Fonseca, Valéria S. Pressões Ambientais e Respostas Estratégicas: Uma Análise de Organizações do Varejo Farmacêutico Curitibano. Revista Eletrônica de Administração, Edição 63, Vol. 15, № 2, 2009.

Hrebiniak, Lawrence G. Making Strategy Work. Philadelphia: Wharton School Publishing. 2005. 
Hitt, Michael A., Ireland, Duane \& Hoskisson, Robert E. Administração Estratégica. $2^{\mathrm{a}}$ Ed. São Paulo: Cengage, 2008.

Lopes, Humberto E. G., Moura, Thelmo N. \& Oliveira, Caio C. G. Os perfis estratégicos no Brasil: Um Estudo dos Agentes Franqueados do Correios a partir da Tipologia de Miles e Snow. RBGN, São Paulo: v. 12, n. 37, p. 388-404, 2010.

Machado-da-silva, Clovis L., Fonseca, Valéria S. da \& Fernandes, Bruno H. R. Mudanças e estratégias nas organizações: perspectiva cognitiva e institucional, Enanpad, Anais... 1998.

Machado-da-silva, Clovis L. \& Vizeu, Fábio. Análise Institucional de práticas Formais de Estratégia. Revista de Administração de Empresas, são Paulo, v. 47, n. 4, 2007.

Martins, Tomas S., Kato, Heitor T., Cruz, June A. W., Reis, Júlio. A. \& El-kouba, Amir. A Influência da Tipologia Estratégica de Miles e Snow no grau de Orientação para o Mercado em Instituições de Ensino Fundamental e Médio do Estado do Paraná. Revistas Gerenciais, v.7, n.2, p 125-137, 2008. São Paulo.

Miles, Raymond E. \& Snow, Charles C. Organization strategy, Structure and process. New York: McGraw-Hill, 1978.

Mintzberg, Henry, Ahlstrand, Bruce \& Lampel, Joseph. Safári de estratégia: um roteiro pela selva do planejamento estratégico. Porto Alegre: Bookman, 2007.

Mintzberg, Henry, Lampel, Joseph, Quinn, James. B. \& Ghoshal, Sumantra.. O Processo da Estratégia: Conceitos, Contexto e Casos Selecionados. Bookman, São Paulo, 2003.
Oliver, Christine. Strategic responses to institutional processes. Academy of Management Review, v. 16, n.1, p. 145-179, 1991.

Oliver, Christine. The antecedents of deinstitutionalization. Organization Studies, v. 13, n. 4, p. 563-588, 1992.

Perola, Andreia C. \& Gimenez, Fernando A. P. Estratégia em pequenas empresas: uma aplicação dos modelos de Miles e Snow e Kirton nas lojas varejistas dos shopping centers de Maringá -PR. Rio de Janeiro: Enanpad, Anais... 2000.

Porter, Michael. E. Estratégia competitiva: técnicas para análise de indústrias e da concorrência. 2.ed. Rio de Janeiro: Campus, 2004.

Ribeiro, Ronaldo, Rossetto, Carlos R. \& Verdinetti, Miguel A., Comportamento estratégico e a visão baseada em recursos: um estudo no setor varejista de material de construção. Gestão e Produção. São Carlos, v.18, n.1, p. 175-192, 2011.

Scott, W. Richard. Instituitions and Organizations. Thousande Oakes: Sage, 2001.

Simon, Herbert. A. Comportamento Administrativo. 2 ed. Rio de Janeiro: FGV, 1970.

Teixeira, Omar R. P., Soares, Marison L. \& Pelissari, Anderson S. Comportamento Organizacional: Uma Aplicação da Tipologia de Miles e Snow no Setor Hoteleiro de Florianópolis. SC. Revista de Administração - UFSM, Santa Maria, v. n.1, p 251267 mai./ago. 2011.

Vasconcelos, Ana C. F., Guedes, Isabela A. \& Cândido, Gesinaldo A. Aplicação dos Modelos de Miles e Snow e Kirton em Pequenas Empresas: um Estudo Exploratório. Gestão da Produção, Operações e Sistemas, Ano 2, v. 3, p. 123- 132, 2007. 